\title{
Evaluating Implementation of The Diabetes Perioperative Passport: A Qualitative Focus Group Study
}

Funke Akiboye ( $\square$ akiboyef@doctors.org.uk)

University Hospital Southampton NHS Foundation Trust https://orcid.org/0000-0002-7632-5587

\section{Emma Page}

Ipswich Hospital

Krish Nirantharakumar

University of Birmingham

Gerry Rayman

Ipswich Hospital

Antje Lindenmeyer

University of Birmingham

Research article

Keywords: us groups, facilitators and barriers, implementation, diabetes, empowerment, passport

Posted Date: July 14th, 2020

DOl: https://doi.org/10.21203/rs.3.rs-40474/v1

License: (c) (i) This work is licensed under a Creative Commons Attribution 4.0 International License.

Read Full License 


\section{Abstract}

Aims To examine the factors affecting implementation of an empowerment tool for patients with diabetes in two surgical groups within a single district general hospital

Background A perioperative passport was developed to improve care of people with diabetes undergoing elective surgery by equipping patients with a hand-held summary of their care needs. Two surgical groups were approached to be involved in developing and piloting this novel technology with contrasting results.

Design A qualitative study using focus groups to examine factors affecting staff engagement in implementing the diabetes perioperative passport.

Methods Two focus group were conducted in 2017 with pre-operative nurses in the departments of orthopaedics and general surgery of an NHS trust $(\mathrm{N}=8)$. Audio-taped group discussions were transcribed, and the data coded and analysed to identify themes.

Results The general surgical nurse group generally supported each other's views and comments. They were enthused about the passport throughout. In contrast, the orthopaedic specialist nurse group had initial reservations about implementing the passport, but as the focus group unfolded, they moved from sharing individual views to finding commonalities and became more engaged and motivated to use the passport.

Conclusion Focus groups are a practical and efficient tool to conduct within the usual working environment of an NHS hospital. In addition to capturing information about the challenges to implementation they may serve as a crucial implementation tool where evidence-based practice has not been adopted.

Impact This is the first study carried out in the NHS setting highlighting the benefit of focus groups as a practical implementation tool.

\section{Contributions To Literature}

- Diabetes is managed in the outpatient setting with care focusing on education and patient empowerment.

- Barriers for implementation of empowerment tools have not been fully explored.

- This study suggests the implementation of empowerment tools may pose an additional challenge to more specialist groups.

- It demonstrates that focus groups are practical and efficient and may also have utility in the implementation process beyond identifying the barriers to embedding a new technology.

- These findings contribute to the small body of evidence on the implementation of empowerment tools and highlight the benefit of focus groups as a useful tool in the implementation process. 


\section{Introduction}

European health care systems are currently facing multiple challenges. People are living longer and together with improvements in care this has resulted in a rising burden of chronic disease. There is now an increasing need for innovation to continue to deliver effective, safe care despite these pressures and limitations.

Diabetes is one such chronic condition which is growing in prevalence, with 3.9 million people diagnosed in the UK; this number is estimated rise to over 5.3 million by $2025(1 ; 2)$. It accounts for $10 \%$ of the NHS budget with a significant portion of these costs related to hospitalisation (3). People with diabetes are more likely to be admitted to hospital both for medical reasons and for surgical procedures than those without the condition (4). The patient journey for surgery from referral, through surgical and preassessment clinics, admission and theatre to recovery and discharge home is complex and involves a range of healthcare professionals. Diabetes adds a further challenge to this perioperative pathway; however no single healthcare professional specifically focuses on diabetes management, despite the poorer outcomes to surgery associated with sub-optimal glucose control (5). There is opportunity for innovations to improve care with potential cost savings. The management of diabetes in the community has moved from a doctor centred to a more individualised and patient centred approach with education and empowerment at its core (6). Education for inpatients with diabetes is usually delivered by diabetes specialist nurses; However, hospitalisation often results in the patients handing over access to food and medication and ultimately their diabetes care to non-specialists as $22 \%$ of hospitals in England and Wales do not currently have dedicated diabetes nurses (7). With 1 in 7 inpatients affected by diabetes, novel ways to provide education to patients and empower them in the hospital setting must be developed (7). It is also crucial that trained non-specialists are able to deliver these innovations.

Even when innovations are evidence based and shown to improve care, their implementation in the NHS can be difficult with variable adoption into routine practice (8). The National Institute for Clinical Excellence (NICE) highlights 5 main barriers to implementation: awareness and knowledge, skills, acceptance and beliefs, motivation and practicalities (9). They stress that staff delivering the care need to be aware of the need for change; they must be trained where necessary to adopt the new technology. In order for this to be successful, they have to believe that they are able to deliver the innovation and that it will make a positive clinical difference and it must be adequately resourced in terms of personnel and time (9).

Despite the pressing need to innovate in the health service, strategies for implementation are inconsistently employed in practice. Furthermore, if implementation is unsuccessful it is not routine practice to evaluate the reasons why to address these factors. However, exploration of the reasons for failure to adopt an innovation could provide the key to integrating a new technology into routine practice.

\section{Background To The Problem}




\section{History of problem}

A novel perioperative passport was designed to empower patients and improve care. This A5 ring-bound folder provides information for patients with diabetes on what to expect at each stage of the perioperative journey and what they could do to help manage their condition. In addition to patient information, the passport has sections for staff to fill in including information relating to their care such as weight, $\mathrm{BP}$ and $\mathrm{HbA} 1 \mathrm{c}$, enabling staff to document key information for the patient and illustrate any differences from the usual targets for these parameters. The aim was for people with diabetes to carry this patient-held record, literally taking their diabetes care in their own hands, to each professional contact from pre-surgical assessment to discharge. Example pages are shown in Box1.

The two largest pre-operative surgical nursing groups in the hospital were approached to participate in developing the passport at its inception and piloting the tool once produced. The project and pilot were enthusiastically taken up by a general surgical group; however, when it came to extending the pilot to a second surgical department in orthopaedics, implementation proved more challenging.

As part of the usual perioperative pathway, both groups assess the safety of patients to proceed with surgery and inform those with diabetes of risks of poor glycaemic control and any adjustments to make to their medication. A diabetes medication information leaflet is among the information both groups can distribute to appropriate patients. Participating nurses were asked to deliver the passport during their existing pre-assessment appointment and use it to provide a structured explanation of the patient's role in their own care, emphasising the importance of good glycaemic control from initial contact to discharge. Observations routinely recorded at this stage such as blood pressure, weight and $\mathrm{HbA} 1 \mathrm{c}$ should also be entered on the passport. At home, the patient could familiarise themselves with what to expect prior to hospitalisation and bring in anything that would help with their glycaemic control around surgery, documenting their usual eating and treatment routines in the passport. Before and after surgery, the passport would be used by ward staff to treat and help patients manage their glucose levels; a discharge checklist empowers the patient to ensure that they are being discharged safely.

The experienced band 8 lead nurse of the general surgical nurse (GSN) group played a key role in the design and development of the passport, ensuring it would be usable and relevant to the broad range of surgical specialities managed by her team. Once developed the passport was presented to the GSN group with guidance and training provided on how to use it in daily practice. Additionally, they were asked as part of the pilot to feed back about its usefulness and content in practice; they were informed we would also ask for feedback from patients. Conversely, the orthopaedic surgical nurse (OSN) group did not participate in developing the passport but were approached again to pilot it with their patient group and provide feedback on its utility.

In order to prepare the GSN group for implementation of the passport, there was a session to present the passport to them and train them in using it attended by around 7 nurses. They were actively encouraged by their service lead to incorporate it into their consultations and shown how this would work in practice. It was more difficult to address the OSN group all together and following initial efforts to involve them in 
development of the passport a number of visits were made to their shared office over the course of several weeks to catch them individually to explain the passport and encourage them to issue it to their patients. Although hospital data indicated that the largest volume of elective surgery on people with diabetes in the trust was carried out in orthopaedics, the OSN group did not manage to hand out the passport to any patients. Following this, the decision was made after several months for the inpatient diabetes specialist nursing team to post the passports out to orthopaedic patients directly with a brief contact phone call explaining how to use it. This enabled patient feedback to be captured from orthopaedic patients.

Initially, we aimed to explore acceptability and feasibility of delivering the passport. An additional research question emerged from the observation of the differing responses to the opportunity to develop and pilot the perioperative passports between the OSN and GSN groups within a single hospital which is the main focus of this article. It is uncommon to have the opportunity to gain feedback from nonresponders in research, however this is crucial for successful implementation of evidenced technologies (10). This study therefore uses focus groups to explore the utility and practicality of implementing the diabetes perioperative passport aiming to extend its use to the OSN group. The groups also form a 'case study' of differences in organisational culture at the same trust and provide an insight into the ways in which these differences can shape the implementation of an intervention. These insights are valuable for the planning and rolling out of interventions throughout the NHS.

\section{Aims/Use of focus groups}

This article focuses on the challenges experienced by orthopaedic specialist nurses in implementing the diabetes perioperative passport in their clinical work in comparison to the group of general surgical preassessment nurses. As these differences were observed within the same trust, an exploration of the groups' differing work cultures and challenges was of greater interest than larger structural barriers which the GSN group had overcome. Qualitative research lends itself well to this research question as it aims to explore the reasons why there were differences in involvement with the passport between the groups.

The focus group methodology involves facilitated discussion between colleagues sharing their views on the passport and enables a collective voice of each of the groups to be heard, drawing out opinions on adopting this initiative in the nurses' respective clinical areas. It was important to note the differences in the structure of the teams and observe the group dynamics that may account for their contrasting responses to the passport. and observe the way participants communicated and interacted to influence each other within the discussion.

\section{Ethical considerations}

An email and written information sheet outlining the aim of the research and assuring participants that their responses would be anonymised was distributed to nurses. To allow both groups to freely voice their opinions we presented patient feedback on the passport and asked for staff to consider whether it would 
work in its current state in their clinical area. This approach was also chosen to minimise a perception of being judged and resultant distress especially for the group who did not implement the passport.

The project received ethical approval from the University of Birmingham.

\section{The researchers' role}

FA worked in the trust as a diabetes research fellow which involved promoting initiatives set up by the diabetes team and collecting data on impact. FA and the passport project manager, EP, met the OSNs individually to show them the passport, invite them to hand it out and reinforce the need for data gathering from their patient group.

EP was involved in developing the passport, promoting and educating nurses on its use and cofacilitating in the focus groups with FA.

\section{Methods}

\section{Participants}

The GSN group comprised 3 participants with 5 OSNs in attendance.

\section{Research site location}

The study was carried out at district general hospital with a sizable orthopaedic department taking elective surgery from the surrounding areas.

\section{Focus groups}

The groups were carried out in each group's respective clinical/ office space for over lunch for comfort, convenience and practicality.

The focus groups were conducted over around 45 minutes with three sections:

\section{Overview and feedback on the passport}

EP presented a brief overview of the challenges for the patient with diabetes in navigating the surgical pathway, highlighting the impetus for developing the passport. Patients' feedback on the passport was presented by PowerPoint; this has been reported separately (11). Participants were free to ask questions.

\section{Reviewing the passport}

Passports were distributed at the beginning of the focus groups giving participants an opportunity to see its layout and finish, and refer to it during the presentation.

\section{Exploring usability and applicability}


The main focus group was conducted using a specifically developed, semi-structured topic guide (see supplement) to explore staff attitudes to the passport exploring whether and how they felt they could incorporate it into their clinical practice.

\section{Analysis}

The focus groups were transcribed, and two coding cycles applied to the transcripts. The first cycle used both in-vivo coding (using participants' own words) and process coding to elicit the nurses' perspectives on the passport and to allow the interaction of individuals to be explored (12). Memos of the researcher's thoughts and observations made during the first coding cycle were fed into the second coding cycle along with the in-vivo and process codes. In the second cycle, the focus was on the dynamics within the group. Therefore, gerunds such as agreeing, reinforcing and questioning increasingly emerged in this phase. This phase integrated first cycle codes and coding of the researcher's memos in order to develop further themes from the data.

The emergent themes were applied to appropriate frameworks. As the themes from the in-vivo coding were largely nurses' understanding of the passport and challenges they faced in implementing it in their department, they were first mapped to a comprehensive summary of the known barriers and facilitators taken from a systematic review on implementation of technologies in the hospital setting (13). Themes from the second phase will be discussed in relation to differences between the two groups and the group dynamics.

\section{Results}

\section{Group structure}

The structure of the GSN and OSN groups differ. The OSN group were experienced nurses (band 6-8) working with a named orthopaedic consultant, their associated anaesthetist and designated secretary. Each OSN therefore specialised in a joint or limb area for example hip and knee. While there may be some cross cover of duties at times, there is no leader or hierarchy within the group of OSNs. The GSNs were a small group of Band 3-5 nurses led by a dynamic and experienced lead nurse on the cusp of retirement who was the point of contact for a wide range of surgical teams and anaesthetists. Regular meetings allowed information, changes and concerns to be cascaded to the more junior nursing team. They all preassess patients from a range of surgical specialities.

The participants represented a pragmatic sample of available pre-assessment nurses on a given day for both groups. The OSN group comprised a significant portion of the whole group with 5 nurses, while a smaller sample of 3 nurses participated from the GSN group. The lead nurse from the GSN group who had been heavily involved in development of the passport had retired at the time of interview and was therefore not in attendance.

\section{Emergent themes}


The themes emerging from the first round of coding the OSN and GSN transcript were mainly barriers and facilitators of implementation such as lack of time. These themes mapped well onto categories from a systematic review of staff reported barriers and facilitators to the implementation hospital-based intervention (13). This provided a valuable framework to display the key areas reported by the nurses during the focus groups in the wider context of implementation within hospitals. The 12 categories identified in the systematic review are displayed, with key domains highlighted in bold within Table 1.

There were:

1. Environmental context, particularly staff workload and time

2. Culture - attitude to change, motivation, champions and role models

3. Staff commitment and attitude- need and ownership

4. Role identity

5. Skills, ability and confidence

\section{Data analysis}

\section{Similarities between groups}

The themes emerging from the groups highlight the perceived strengths and weaknesses of the passport from a nursing perspective and identify the facilitators and barriers to its implementation in both preassessment clinics.

The areas the nurses in both groups expressed similar views or approaches on the passport were:

- Appreciation of benefit of passport to patients

- Importance of an up to date and comprehensive document

Quotes illustrating these areas of agreement are shown in table 2.

\section{Differences between groups}

Despite universal appreciation of the passport as beneficial and a well-designed tool, there were areas in which the groups differed in their willingness to utilise it in their routine practice.

These centred on the work required and the nurses' ability to find time and have the resources to implement it in practice. Contrasting attitudes are highlighted in in table 2.

There were 4 key areas where groups differed:

- Ease of use/ time burden

- Culture and attitude to change

- Staff role identity 
- Skills/ ability/ confidence

\section{Ease of use/ time burden}

The GSN group did not feel that the passport significantly added to their workload while the OSNs expressed that this would be a duplication of some information they had in leaflets for patients with diabetes. Some felt that the additional explanations and documentation of observations in the passport could be time-consuming and patients with diabetes would therefore require longer appointments.

\section{Culture and attitude to change}

There also were differences in culture between the groups and among individuals in the OSN group in their attitude to change. The GSN group maintained their senior nurse's openness to change as shown by her involvement in developing the passport and encouraging staff to use it. In the OSN group a nurse who offered to hand them out to her patients initially was a lone voice in the focus group.

\section{Staff role identity}

\section{Advocacy vs Authority}

There is a contrast between the two nursing groups in the roles the nurses played in supporting the patient journey. The advocacy role is more evident from the outset with the GSN nurses.

In care settings, advocacy is a process of supporting and enabling people to express their views and concerns, access information and services, explore their options and promote their responsibilities (14). GSNs spoke a lot from a hypothetical patient perspective "if I was a patient..." and portrayed the passport as useful and informative for patients. This perhaps reflects their junior level and consequently the more limited experience they could draw on. Their role is not to explain the surgery in detail to the patient, but rather prepare them for it, giving patients an idea what to expect in hospital and signposting where necessary. It is worth noting that one of the nurses in the GSN focus group was a staff nurse with type 1 diabetes. She had not used the passport but spoke enthusiastically, sometimes from a personal viewpoint, of living with the condition and expressed she felt it was a good idea for patients. Nurses drew on empathic advocacy about patients being the expert of their disease which was likely influenced by her presence: "they're the best people to manage their condition, aren't they".

The OSN group nurses had specialist knowledge in their limb or joint area and the information they give patients is tailored with some pathways structured slightly differently. They also describe a way of working which is specific to the consultant(s) they work with; consequently, information can be provided not only about the latest evidence for that type of surgery, but also about the operating surgeon's and anaesthetist's usual practice. "we're going to be giving our hip and knee patients a high carb drink to take home... on the enhanced recovery programme". This implied a different approach to patients attending pre-assessment. They take a more directive approach, informing patients what to expect and enforcing what was required of them. They appeared to carry more responsibility for ensuring perioperative 
readiness and had the power to delay surgery if this was not delivered. "... when I saw him at preadmission I told him that if his ...HbA1c weren't better... we couldn't do surgery". Taking on this authoritative role is challenging at times: "you don't want to tell them they need to lose loads of weight..."

Later in the focus group discussion the OSNs also mentioned their role as patient advocates. This is 35 minutes into the group compared with just 6 minutes for the GSN's, who alluded to this role enthusiastically and frequently.

\section{Skills, ability, confidence}

The OSN group suggested that the passport was a good idea; however there was a large perceived impact on their time and workload. This is in contrast to the GSN group who felt it was quick and easy to use in everyday practice. This difference may have, in part, been a reflection of lower confidence they had in explaining diabetes care to patients in the detail that the passport appeared to require.

\section{Changes during focus group}

During the process of coding, differences in responses between the groups became apparent. Additionally, there were some themes where OSNs as a group appeared to change their position as the focus group progressed. The domains of the barriers and facilitators framework where these changes were most marked were motivation and group identity.

\section{Motivation}

While the OSN group appreciated it was a useful intervention for patients, this statement was generally caveated by the question of what would be expected of them in implementing it emphasising that this would affect their workload. This perception appeared to shift after one of the nurses shared a story of a personal challenge with an aggressive patient whose surgery had to be delayed due to poor glucose control. The personal benefit to the nurses of using the passport was illustrated and there were no further objections to its utility.

\section{Team identity}

The structural differences in the set-up of the two nursing groups is apparent early in their interactions. The GSNs were speaking as peers doing the same work who could ask each other for advice and support. They shared practice and affirmed each other's statements and observations. The GSN group were quite vocal in their comradery making affirmative comments and statements throughout.

Initially, the OSN nurses spoke about their individual joint or limb practice or quirks of the surgeon they worked closely with, which did not relate to the practice of others in the group. They gave each other space to speak and respected the position of their peers, but were not seeking to support each other through the initial interactions. As the focus group progressed, the OSN group appeared to become more cohesive in emphasising and expanding on what their colleagues had just stated. 
There was a noticeable difference towards the latter part of the OSN focus group. The pivot point appeared to be strong words from a senior nurse about the workload and time pressures. She expressed a collective will to help patients, but also the shared feeling of time scarcity. With this clearly and openly stated the nurses appeared able to move past their individual objections to the passport and started brainstorming how it may be implemented at other stages in the pathway to allow patients to benefit whilst limiting the impact on their own workload: "we do a hip and knee group, perhaps you could come and hand it out there".

The behaviour change model helps categorise what is observed during the focus group. The main change appears to be centred around scenario-based risk taking. Over the course of the discussion the nurses in both groups offered issues they had encountered or noticed themselves with patient care. Finally, the single story shared in the OSN group around this challenging patient scenario was an act of storytelling or Scenario-based risk-taking. It was powerful for both groups in connecting them as a team with shared experiences and particularly with the OSN group helped address their collective concerns and provided a potential individual motivation to use the passport.

\section{Discussion}

People with diabetes are educated and empowered to facilitate daily self-care to manage their condition. This patient-held booklet encourages patients to retain ownership of their condition whileencountering a range of professionals throughout their perioperative journey.

Our findings indicate that although two groups within the same organisation may share positive views about a new intervention, the attitude to and subsequent success of implementation is highly dependent on other factors. Crucial barriers were noted under the sub-domains of staff commitment and attitudes to change, role identity and skills, ability and confidence with differences in the perceived extra workload or time commitment required to administer the passport. The reasons for this contrast appears to, in part, be related to differences in the composition and structure of the teams.

The group structure and presence of a team with a shared purpose and clear leadership is known to be an important factor in implementation (15). Change orientated leadership has been shown to aid learning and psychological safety within a team (16). While the GSNs benefited from this in their group structure: the OSN group worked alongside their consultants and so did not; this structure also appeared to link to their professional identity.

In the GSN group staff had a role identity as patient advocate which fed into the belief that the passport addressed patient needs and that they could reasonably incorporate it into their practice. For the OSNs, the empowering purpose of the passport was perhaps more challenging to the existing role identity of a more specialised position; they also required additional time to incorporate it into their role.

The place of professional role identity in the implementation of empowerment tools has not previously been explored in the literature. However, a study by Dijkstra and colleagues noted more resistance 
embedding an empowerment tool among consultant physicians compared to diabetes nurses (17). There were mixed views among the consultant group with lack of motivation and lack of time cited as barriers to implementation in keeping with our study, perhaps indicating that they identified their value as their expertise and specialist knowledge in a similar way to the OSN group. The differing response of the staff groups to this tool suggests that role identity can act as either a barrier or facilitator (13).

Conversely, rather than having fixed identities, staff can also take on a range of roles, for example in achieving patient adherence. Following a year-long ethnographic study roles adopted by doctors in treating patients with diabetes were categorised as educators, detectives, negotiators, salesmen, cheerleaders and policemen depending on how they interacted with the patient and the strategies they employed to encourage adherence e.g. praising for achievement of goals or warning of complications (18). It is interesting to note the more authoritarian style of the OSN group nurses is in line with the "policeman" role Lutfey describes. This is in contrast to the more "cheerleading" role taken by the GSNs. This observation raises the question of the role adopted by the person delivering an intervention in the success of implementation including the role of a group's collective dominant working style. The most effective style of implementing in each group may be influenced by the dominant style they adopt in clinical practice. It may be that HCPs groups who have a predominantly authoritative or "policeman" way of working respond best to the same style they adopt or a very contrasting style such as negotiator or detective whereas cheerleaders, who already share a collective agenda, might respond best to a policemen approach. This is a potential area for further exploration.

Beyond highlighting the dynamics of the groups, perhaps the most interesting finding in this study was the role of the focus group in facilitating change, which is otherwise poorly described in the literature. Despite definitions of focus groups as opportunities to observe the interaction between participants and the shared development of thoughts and ideas is rarely reported. Instead, researchers appear to use them as time efficient simultaneous individual interviews. This discrepancy has been explored in a metaanalysis by Belziles et al which explains the key role of group cohesion in this process. "If participants manage to establish common ground they behave more as a small group (co-creating the narrative as they go along);if they do not...they will behave more as individuals "expressing private views (19). This again highlights the importance of the differences in structure of the groups, but goes some way to explaining the place of story-telling in producing these changes in the OSN group during the focus group. The power of story-telling was also noted in some studies included in Geerlig's review where sharing success stories influenced in staff's readiness for change (13). Importantly, these findings reinforce the potential usefulness of focus groups not only in gathering information about the barriers for implementation, but additionally and crucially as a behaviour change tool in their own right.

\section{Reflections}

The primary researcher FA was promoting the passport with nurses and in this role recognises that codes may have been assigned to the two groups reflecting some of the resistance encountered from the OSN group prior to the focus group. There did not appear to be a particular anxiety in the group about 
speaking openly however as many in the group had previously been approached directly there may have been some initial guarding in their responses.

The observation of the emerging narrative between focus group participants is thought to originate from the researcher's assumption that the participants co-construct meaning rather than participants sharing their pre-formed opinions. This approach is associated with more socially-orientated researchers (19).

Strengths and Limitations

The study is limited in the size of the GSN group which had only 3 participants for practical reasons. Although this is less than usual for a focus group it is not likely to have had a significant impact on the view shared as the participants in this group had a very similar approach.

There were 5 participants in the OSN group which was appropriate as a larger group may have silenced some colleagues from active participation. In both groups every participant contributed to the discussion.

The senior nurse who set up the passport had since retired and was not therefore involved in the focus groups which allowed the small group of GSNs to speak freely about their views on the passport.

\section{Conclusions And Implications}

The data shows that evidence-based tools can be challenging to implement within a department even if the tool is regarded by staff as beneficial for patients. Perceived lack of time and motivation are barriers to the implementation of empowerment tools which may be more notable in staff with specialist and authoritative role identities. However, this may be modified by addressing other areas such as skill and patient related barriers.

Another useful finding is related to the use of focus groups. They are routinely conducted to assess the barriers implementing an evidence-based technology in a time pressured and busy hospital setting. Moreover, through sharing of stories or scenario-based risk taking, focus groups can be vehicle for behaviour change and may aid implementation in areas or departments where adoption of a tool has previously been less successful. The ways in which professionals involved in implementation in the healthcare setting tailor their strategy to different groups is a potential area for further research.

\section{Declarations}

\section{Author contributions}

FA and KN developed the concept of the study with input from AL. FA designed the study with supervision of AL. FA carried out the focus group, transcribed and analysed the data and prepared the manuscript under the supervision of AL. EP was involved conducting the focus groups and the designing and implementation of the passport with FA and under the guidance and close supervision GR. All authors have read and approved the final manuscript. 


\section{Competing interests}

The authors have no conflicts of interests. No external funding was received to conduct this research.

\section{Ethics approval and consent to participate}

This research was approved by University of Birmingham's ethics committee and written consent was gained from participants.

\section{Funding}

No funding was received for this research

\section{Consent to publication}

Not applicable to this research.

\section{Availability of data and materials}

Transcripts are not available in public repository for participant confidentiality, but portions are available on reasonable request from the first author.

\section{Acknowledgements}

Thank you to the Diabetes Specialist Nursing team Rachael Marie-Round Christopher Kerry, Yasmin Hunt, Sarah Barker and the DICE team for their contribution to developing and distributing the perioperative passport.

\section{Abbreviations}

OSN - Orthopaedic specialist nurse

GSN - General surgical nurse

HCP - Health care professional

\section{References}

1. Diabetes UK. Diabetes Prevalence 2019. [Online] 2019. [Cited: 29 Feburary 2020.] https://www.diabetes.org.uk/professionals/position-statements-reports/statistics/diabetesprevalence-2019.

2. Number of people with diabetes reaches 4.8 million. [Online] 2020. [Cited: 29 February 2020.] https://www.diabetes.org.uk/about_us/news/diabetes-prevalence-2019. 
3. The cost of diabetes. [Online] 2014. [Cited: 29 February 2020.] https://www.diabetes.org.uk/resources-s3/2017-

11/diabetes\%20uk\%20cost\%20of\%20diabetes\%20report.pdf.

4. Comino E J, Harris M F and Haas M. Impact of diabetes on hospital admission and length of stay among a general population aged 45 year or more: a record linkage study. 2015, BMC Health Services Research, Vol. 15, p. 12.

5. S Serio, J M Clements, D Grauf and A M Merchant. Outcomes of Diabetic and Nondiabetic Patients Undergoing General and Vascular Surgery. 963930, 26 December 2013, ISRN Surg.

6. Boström E, Isaksson U, Lundman B, Lehuluante A, Hörnsten Å. Patient-centred care in type 2 diabetes - an altered professional role for diabetes specialist nurses.. 4, s.I. : Nodric college of science, 2014, Scand J Caring Science, Vol. 28, pp. 675-82.

7. NHS digital. National Diabetes Inpatient Audit 2018. s.I. : NHS digital, 2019.

8. NHS Innovation Accelorator: The AHSN Network. Understnading how and why the NHS adopts innovation:: The Bayswater Institute, 2018.

9. National Institute for Health and Clinical Excellence. How to change practice - understand, identy and overcome barriers to change. 2007.

10. The impact of non-repsonders on health and lifestyle outcomes in an intervention study. Hansen $\mathrm{E}$, Fonager K, Freund KS, Lous J. 632, 2014, Vol. 7.

11. Page E, Akiboye F, Jackson S, Kerry C, Round C, Rayman G. Perioperative passport: empowering people with diabetes along their surgical journey. 12, 2017, Vol. 34, pp. 1737-1741.

12. Saldana J. The Coding Manual for Qualitative Researchers. s.I. : SAGE Publications Ltd, 2016. IBSN 978-1-4739-0248-0.

13. Hospital based interventions: a systematic review of barriers and facillitators into the implementation process. Geerligs L, Rankin NM, Shepherd H, Botow P. 13, 2018, Implementation science, Vol. 36.

14. Carlisle S. Health promotion, advocacy and health inequalities: a conceptual framework. Health Promotion International, 2000, Vol. 15, pp. 369-76.

15. Ortega A, Van den Bossche P, Sánchez-Manzanares M, Rico R \& Gil F . The Influence of ChangeOriented Leadership and Psychological Safety on Team Learning in Healthcare Teams. Journal of Business psychology 2014, Vol. 29, pp. 311-321.

16. Dijkstra R, Braspenning J, Grol R. Empowering patients: how to implement a diabetes passport in hospital care. 2, 30 October 2001, Patient Education and Counseling, Vol. 47, pp. 742-747.

17. Lutfey K. On practices of 'good doctoring': reconsidering the relationship between provider roles and patient adherence. 4, 2005, Vol. 27, pp. 421-447.

18. Belzize J, Oberg G. Where to begin? Grappling with how to use particiapant interraction in focus group design. Qualitative reserach, 2012, Vol. 12, pp. 459-472. 


\section{Tables}

Table 1.

This table summarises the broad categories (system, staff and environment) and areas within these categories found to be barriers and facilitators to implementation in a recent systematic review (13). The subdomains highlighted in this study are indicated in bold.

\begin{tabular}{|c|c|c|}
\hline & Domain and description & sub-domain examples \\
\hline \multicolumn{3}{|l|}{ SYSTEM } \\
\hline & $\begin{array}{l}\text { Environmental context } \\
\text { Physical, structural resources of the } \\
\text { context, along with its processes and } \\
\text { personal resources }\end{array}$ & IT, staff, time, workload \\
\hline & $\begin{array}{l}\text { Culture } \\
\text { System culture, beliefs and behaviours } \\
\text { in relation to change and staffing roles }\end{array}$ & $\begin{array}{l}\text { Attitude to change, commitment and } \\
\text { motivation, flexibility of roles/trust, } \\
\text { champions/role models }\end{array}$ \\
\hline & $\begin{array}{l}\text { Communication processes } \\
\text { Online and in-person communication } \\
\text { methods }\end{array}$ & Processes within the context \\
\hline & $\begin{array}{l}\text { External requirements } \\
\text { External pressures or expectations }\end{array}$ & Reporting, standards, guidelines \\
\hline \multicolumn{3}{|l|}{ STAFF } \\
\hline & $\begin{array}{l}\text { Staff commitment and attitudes micro- } \\
\text { level beliefs, attitudes and behaviours } \\
\text { toward change in general, and the } \\
\text { intervention }\end{array}$ & $\begin{array}{l}\text { Perceived validity/need, ownership, } \\
\text { perceived efficiency of the intervention }\end{array}$ \\
\hline & $\begin{array}{l}\text { Understanding/ awareness } \\
\text { Understanding the aims and } \\
\text { methodology of the intervention }\end{array}$ & $\begin{array}{l}\text { Understanding the goals of the intervention, } \\
\text { and of the processes/mechanics }\end{array}$ \\
\hline & $\begin{array}{l}\text { Role identity } \\
\text { Beliefs and attitudes towards work role } \\
\text { and responsibilities }\end{array}$ & Flexibility, responsibility \\
\hline & $\begin{array}{l}\text { Skills, ability, confidence } \\
\text { Sense of capacity to carry out the tasks } \\
\text { of the intervention, managing barriers } \\
\text { posed by the target population and } \\
\text { their work environment }\end{array}$ & $\begin{array}{l}\text { Ability andconfidence to engage patients, to } \\
\text { carry out the intervention, to manage } \\
\text { stress/competing priorities/ patient barriers }\end{array}$ \\
\hline \multicolumn{3}{|l|}{ INTERVENTION } \\
\hline & $\begin{array}{l}\text { Ease of integration } \\
\text { How well the intervention "fits" with } \\
\text { the current system, resources and } \\
\text { needs of the population and context }\end{array}$ & $\begin{array}{l}\text { Complexity, cost and resources required } \\
\text { acceptability/suitability to system, staff and } \\
\text { patients }\end{array}$ \\
\hline
\end{tabular}




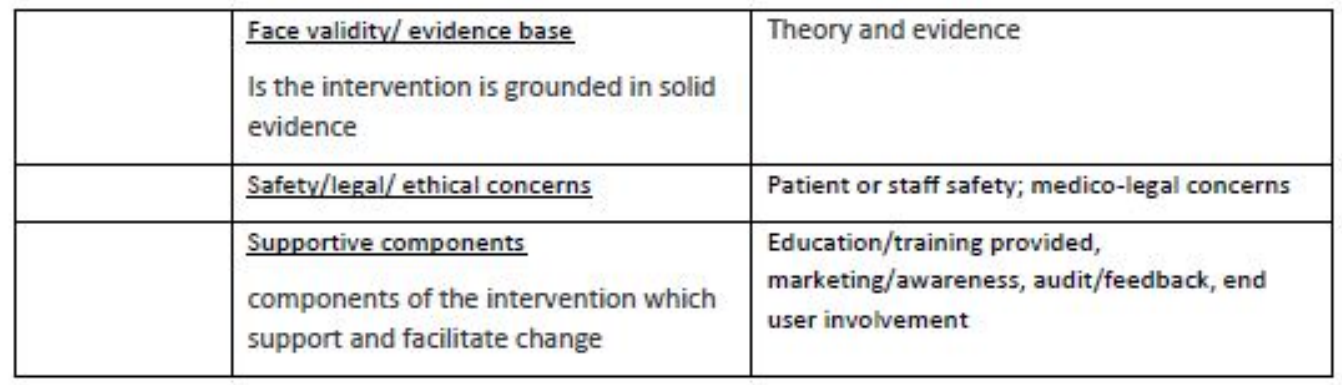

Illustrative quotes for the barriers and facilitators expressed in both groups are displayed in the categories and subdomains they mapped to in table 2 .

Table 2 Mapped illustrative quotes

\begin{tabular}{|c|c|}
\hline Factor & Illustrative quotes \\
\hline \multicolumn{2}{|l|}{ SYSTEM } \\
\hline Environmental context & $\begin{array}{l}\text { Workload: } \\
\text { GSN- if we give it [passport] to all diabetic patients...I would imagine } \\
\text { there's quite a few..- } \\
\text { OSN- It would be every diabetic patient... } \\
\text { OSN- we would have to fill this in as well as our charts... it will take a little } \\
\text { bit more time to do that... } \\
\text { Time: } \\
\text { GSN- We're limited for time and I don't think this [passport] takes up a } \\
\text { huge chunk of time. } \\
\text { OSN- we really, really need to know who the diabetic patients are so we } \\
\text { could have an extra } 10 \text { minutes. }\end{array}$ \\
\hline Culture & $\begin{array}{l}\text { Attitude toward change: } \\
\text { OSN- Is it [passport] more work...that's the main stumbling block with } \\
\text { introducing anything new. } \\
\text { System level commitment: } \\
\text { OSN- he [surgeon] is so inflexible...he's a lovely man, but inflexible. } \\
\text { Champions: } \\
\text { GSN-It would be nice if other departments got to know about it } \\
\text { [passport]... if we just start it up... }\end{array}$ \\
\hline
\end{tabular}




\begin{tabular}{|c|c|}
\hline \multicolumn{2}{|l|}{ STAFF } \\
\hline Staff commitment and attitude & $\begin{array}{l}\text { Attitude toward the intervention: } \\
\text { GSN- Better than what we've got [i.e. generic leaflets], yes by far...more } \\
\text { attractive as well... } \\
\text { OSN- To be honest this [passport] is all we need to give them... } \\
\text { Beliefs regarding the need for intervention: } \\
\text { GNS- We often used to get comments like "how will my diabetes be } \\
\text { managed, you know, while l'm in hospital." } \\
\text { Motivation: } \\
\text { GSN- I'm eager to do one [give out/ explain the passport] with someone } \\
\text { with diabetes staying overnight 'cause I've got type } 1 \text { diabetes as well. } \\
\text { GSN- Anything that's gonna help... to improve or stabilise their condition. } \\
\text { OSN-[nurse gives example of a patient who shouted at her after she } \\
\text { called to delay his surgery due to sub-optimal glycaemic control and felt } \\
\text { written guidance for him would have supported her in this task.] } \\
\text { "When I phone him her was really aggressive. Actually if he had this } \\
\text { booklet.." } \\
\text { "it's good for us as well." } \\
\text { Ownership: } \\
\text { OSN-As long as the nurses on the ward know about it [passport] ... } \\
\text { it's going to go. }\end{array}$ \\
\hline Role identity & $\begin{array}{l}\text { Staff role identity: } \\
\text { OSN-Who are you expecting to do the diabetes check list? }\end{array}$ \\
\hline Skills, ability, confidence & $\begin{array}{l}\text { Confidence to deliver the passport: } \\
\text { OSN- They would have questions... you can't say don't ask me any } \\
\text { questions } \\
\text { GSN- Quite easy for us to do our bit [complete the health professional } \\
\text { sections of the passport] } \\
\text { GSN-...it's all there already so you're literally just taking the patient } \\
\text { through. }\end{array}$ \\
\hline
\end{tabular}




\begin{tabular}{|l|l|}
\hline & $\begin{array}{l}\text { Patient related barriers: } \\
\text { OSN-...if you put too much stuff in they just stop reading it. } \\
\text { GSN-...they would only have this themselves...it's how reliable the } \\
\text { patient is ... on the day...so it's trying to make sure the two are } \\
{[\ldots \text { coordinated]. }}\end{array}$ \\
$\begin{array}{l}\text { Time management: } \\
\text { OSN-We would have to fill this out as well as our charts [pause] which is } \\
\text { fine... }\end{array}$
\end{tabular}

\section{Supplementary Files}

This is a list of supplementary files associated with this preprint. Click to download.

- SRQRchecklistPassport1.1.docx

- TopicguideforpassportfocusgroupforHSR.docx 